
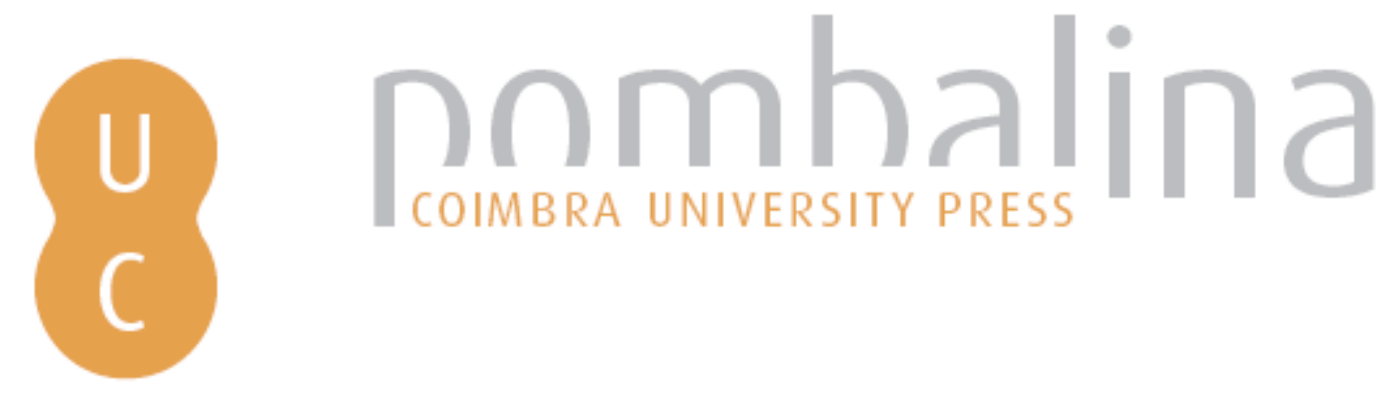

\title{
An upper non-reflecting boundary condition for atmospheric compressible flow
}

Autor(es): $\quad$ Costes, Aurélien; Lac, Christine; Masson, Valéry; Rochoux, Mélanie

Publicado por: Imprensa da Universidade de Coimbra

URL

persistente: URI:http://hdl.handle.net/10316.2/44616

DOI: $\quad$ DOI:https://doi.org/10.14195/978-989-26-16-506_99

Accessed : $\quad$ 26-Apr-2023 12:29:30

A navegação consulta e descarregamento dos títulos inseridos nas Bibliotecas Digitais UC Digitalis, UC Pombalina e UC Impactum, pressupõem a aceitação plena e sem reservas dos Termos e Condições de Uso destas Bibliotecas Digitais, disponíveis em https://digitalis.uc.pt/pt-pt/termos.

Conforme exposto nos referidos Termos e Condições de Uso, o descarregamento de títulos de acesso restrito requer uma licença válida de autorização devendo o utilizador aceder ao(s) documento(s) a partir de um endereço de IP da instituição detentora da supramencionada licença.

Ao utilizador é apenas permitido o descarregamento para uso pessoal, pelo que o emprego do(s) título(s) descarregado(s) para outro fim, designadamente comercial, carece de autorização do respetivo autor ou editor da obra.

Na medida em que todas as obras da UC Digitalis se encontram protegidas pelo Código do Direito de Autor e Direitos Conexos e demais legislação aplicável, toda a cópia, parcial ou total, deste documento, nos casos em que é legalmente admitida, deverá conter ou fazer-se acompanhar por este aviso. 


\section{ADVANCES IN}

\section{FOREST FIRE RESEARCH}

\section{8}

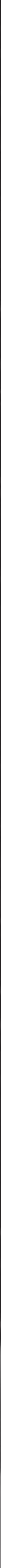




\title{
An upper non-reflecting boundary condition for atmospheric compressible flow
}

\author{
Aurélien Costes* ${ }^{1}$; Christine Lac ${ }^{2}$; Valéry Masson ${ }^{2}$; Mélanie Rochoux ${ }^{3}$ \\ ${ }^{1}$ CERFACS, CNRM, Toulouse, France. \{aurelien.costes@meteo.fr*\} \\ ${ }^{2}$ CNRM, Toulouse,France. \{christine.lac@meteo.fr, valery.masson@meteo.fr\} \\ ${ }^{3}$ CERFACS, Toulouse, France. \{melanie.rochoux@cerfacs.fr
}

\begin{abstract}
So as to predict fire spread after its ignition, the Meso-NH atmospheric model has been coupled to the forest fire model ForeFire. Meso-NH uses the anelastic hypothesis that provides an intrinsic acoustic filter. Nevertheless, this approximation does not allow horizontal density variations which leads to problems close to the fire front because of the high heat release. That is why the full Euler compressible system shall be used to take into account air expansion at the fire level. This governing equations system transition comes with acoustic waves propagation. Although those waves are uninteresting for meteorology, they bounce on the ground and on the roof of the domain and disturb the inner solution. Indeed, the actual rigid lid as upper boundary condition does not allow acoustic waves radiation. The Rayleigh top damping layer already implemented in Meso-NH provides high frequency filtering, meaning that the new upper boundary shall only evacuate the remaining low frequency waves.

In order to build a reliable and precise upper boundary condition for a reasonable computational cost, we shall verify that the new boundary (1) lets low frequency waves pass through and (2) does not force the interior domain state. So as to preserve the scalability of the code, the boundary shall also (3) be localized in space and (4) as localized as possible in time to limit memory storage.

Several methods could be used to evacuate acoustic waves from the domain. Our requirements have conducted us to base our new upper boundary condition for Meso-NH on Characteristic-based Boundary Conditions, known as NSCBC, proposed by Poinsot and Lele. This boundary condition (BC) is known to be "non-reflecting" and also local in space and time. Nevertheless, the NSCBC formulation based on the characteristic theory of Thompson, comes out as "partially non-reflecting" as its behavior is more a low pass filter. An extended method called Plane Wave Masking, PWM, has been proposed by Polifke et al. to get a near zero reflexion coefficient for low frequency waves.

In PWM theory, the acoustic waves are assumed plane and incoming with normal incidence at the boundary. Nevertheless, the normal incidence of wave represents a strong limitation of the formulation. Lodato et al. have built a 3D formulation of NSCBC that computes transverse gradient to take into account oblique waves.

In purpose of testing the PWM formulation for its requirements, an offline model has been created in Python. This simplified model has been used to check the PWM boundary behavior facing plane waves and spherical waves. Once the PWM method has been proved as compliant to each requirement (1-4), it is implemented in the Meso-NH compressible code. Then, different academic and classic Meso-NH cases are run to ensure the correct behavior of the new boundary as orographic waves as well as fire experiments. The compressible assumption will be evaluated with the FireFlux experience.
\end{abstract}

Keywords: Acoustics, Boundary conditions, Non-reflecting, Meso-NH, Compressible, Computational Fluid Dynamics

\section{Introduction}

So as to predict fire spread after its ignition, the Meso-NH atmospheric model (Lafore et al. 1998, Lac et al. 2018) has been coupled to the forest fire model ForeFire (Filippi et al. 2009). Meso-NH uses the anelastic hypothesis that provides an intrinsic acoustic filter. Nevertheless, this approximation does 
not allow horizontal density variations which leads to problems close to the fire front because of the high heat release. That is why the full Euler compressible system shall be used to take into account air expansion at the fire level. This governing equations system transition comes with acoustic waves propagation. Although those waves are uninteresting for meteorology, they bounce on the ground and on the roof of the domain and disturb the inner solution. Indeed, the actual rigid lid as upper boundary condition does not allow acoustic waves radiation. The Rayleigh top damping layer already implemented in Meso-NH provides high frequency filtering, meaning that the new upper boundary shall only evacuate the remaining low frequency waves.

In order to build a reliable and precise upper boundary condition for a reasonable computational cost, we shall verify that the new boundary (a) lets low frequency waves pass through and (b) does not force the interior domain state. So as to preserve the scalability of the code, the boundary shall (c) be localized in space and (d) also localized in time to limit memory storage.

Several methods could be used to evacuate acoustic waves from the domain as mentioned by Colonius (2004). Our requirements have conducted us to base our new upper boundary condition for Meso-NH on Characteristic-based Boundary Conditions, known as NSCBC, proposed by Poinsot and Lele (1992). This boundary condition (BC) is known to be "non-reflecting" and also local in space and time. Nevertheless, the NSCBC formulation based on the characteristic theory of Thompson (1987), comes out as "partially non-reflecting" as its behavior is more a low pass filter as shown in Selle et al. (2004). An extended method called Plane Wave Masking, PWM, has been proposed by Polifke et al. (2006), to get a near zero reflexion coefficient for low frequency waves.

\section{Boundary formulation based on characteristic analysis}

The new boundary is built on the full Euler compressible system ignoring Coriolis and subgrid effects as well as the atmosphere is considered dry, i.e. the mixing ratio of total water substance is null. Those equations used in the compressible version of MesoNH are given by Eq. (2-4). The characteristic study of this system is based on Thomson 1987 analysis which require to write the system of equations as

$$
\begin{aligned}
\frac{\partial \underline{\tilde{U}}}{\partial t}+\frac{\partial \underline{F}_{x}}{\partial x}+\frac{\partial \underline{F}_{y}}{\partial y}+\frac{\partial \underline{F} z}{\partial z}+\underline{D}^{\prime} & =\underline{0} . \\
\frac{\partial \rho_{d}}{\partial t}+\frac{\partial \rho_{d} u_{i}}{\partial x_{i}} & =0 \\
\frac{\partial \rho_{d} u_{i}}{\partial t}+\frac{\partial \rho_{d} u_{i} u_{j}}{\partial x_{j}}+\frac{\partial P}{\partial x_{i}}+\rho_{d} g \delta_{i 3} & =0 \\
\frac{\partial \rho_{d} \theta}{\partial t}+\frac{\partial \rho_{d} \theta u_{i}}{\partial x_{i}} & =0
\end{aligned}
$$

In Eq. (1), $\underline{U}$ is the vector of conservative variables, $F$ the flux vectors and $D^{\prime}$ an inhomogeneous term not containing derivatives. Another form of the system uses the vector of primitive variables $\underline{U}$ chosen as

$$
\underline{U}=\left[\begin{array}{lllll}
\rho_{d} & u & v & w & \theta
\end{array}\right]^{T} .
$$

One can rewrite the Eq. (1) with the new vector of variables.

$$
\frac{\partial \underline{U}}{\partial t}+\underline{\underline{A}} \frac{\partial \underline{U}}{\partial x}+\underline{\underline{B}} \frac{\partial \underline{U}}{\partial y}+\underline{\underline{C}} \frac{\partial \underline{U}}{\partial z}+\underline{D}=0
$$


Then, matrix $A, B$ and $C$ can be diagonalized and lead to

$\frac{\partial \underline{U}}{\partial t}+\underline{\underline{R}} \underline{\underline{L}} \underline{\underline{R}}^{-1} \frac{\partial \underline{U}}{\partial x}+\underline{\underline{S}} \underline{\underline{M}} \underline{\underline{S}}^{-1} \frac{\partial \underline{U}}{\partial y}+\underline{\underline{T}} \underline{\underline{N}} \underline{\underline{T}}^{-1} \frac{\partial \underline{U}}{\partial z}+\underline{D}=\underline{0}$,

with $L, M$ and $N$ diagonal matrix. Let us identify waves as the following terms

$\underline{\mathcal{L}}=\underline{\underline{L}} \underline{\underline{R}}^{-1} \frac{\partial \underline{U}}{\partial x}, \quad \underline{\mathcal{M}}=\underline{\underline{M}} \underline{\underline{S}}^{-1} \frac{\partial \underline{U}}{\partial y}, \quad \underline{\mathcal{N}}=\underline{\underline{N}} \underline{\underline{T}}^{-1} \frac{\partial \underline{U}}{\partial z}$.

For the following, we will focus only on $z$ direction as this is the normal direction of the upper boundary. The characteristic study gives the waves amplitude as a function of normal spacial derivatives.

$$
\begin{aligned}
& \mathcal{N}_{1}=\frac{w-c}{2}\left[\frac{\partial \rho_{d}}{\partial z}-\frac{\rho_{d}}{c} \frac{\partial w}{\partial z}+\varphi \frac{\partial \theta}{\partial z}\right] \\
& \mathcal{N}_{2}=\frac{w+c}{2}\left[\frac{\partial \rho_{d}}{\partial z}+\frac{\rho_{d}}{c} \frac{\partial w}{\partial z}+\varphi \frac{\partial \theta}{\partial z}\right] \\
& \mathcal{N}_{3}=-w \varphi \frac{\partial \theta}{\partial z} \\
& \mathcal{N}_{4}=\frac{w}{2} \frac{\partial u}{\partial z} \\
& \mathcal{N}_{5}=\frac{w}{2} \frac{\partial v}{\partial z}
\end{aligned}
$$

with $\varphi=\frac{\rho_{d}}{\gamma \theta}, u, v, w$ the three components of the wind, $c$ the sound speed defined as $c^{2}=\frac{\gamma P}{\rho_{d}}, \gamma$ the adiabatic index, $P$ the pressure and $\theta$ the potential temperature. The terms described above are the amplitudes of characteristic waves associated with each characteristic velocity as eigenvalues of the matrix $A, B$ and $C$. The first wave moves at the speed of sound $(w-c)$ in the negative $z$ direction it "comes in" the domain at the boundary (Fig. 1). The second one travels also at the speed of sound $(w+c)$ but in the positive $z$ direction. Then, the third characteristic wave is associated to the convective velocity which is the speed of the entropy waves and finally, the last waves correspond to the advection by the vertical wind $w$. At the upper boundary the spacial derivatives can be computed by uncentered schemes and the other variables are available locally which is compliant with our constraints (c).

The time integration used is a simple Forward In Time (FIT) scheme which leads to the following system.

$$
\begin{aligned}
& \underline{U}^{n+1}=\underline{U}^{n}-\Delta t\left[\underline{\underline{T}}^{n} \underline{\mathcal{N}}^{n}+\underline{D}^{n}\right], \text { Poinsot and Lele (1992) } \\
& \underline{U}^{n+1}=\underline{U}^{n}-\Delta t\left[\underline{\underline{R}}^{n} \underline{\mathcal{L}}^{n}+\underline{\underline{S}}^{n} \underline{\mathcal{M}}^{n}+\underline{\underline{T}}^{n} \underline{\mathcal{N}}^{n}+\underline{D}^{n}\right], \text { Lodato et al. (2008) }
\end{aligned}
$$

The exponent $n$ means at time $t^{n}=n \Delta t$ and so $n+1$ is the next time step $\Delta t$. The method used by Poinsot and Lele (1992) is one-dimensional and considers only the normal direction informations to compute the boundary condition. Like most of the non-reflective boundary conditions, Poinsot and Lele (1992) assume that the flow at the boundary can be viewed as locally one-dimensional and aligned with the boundary normal direction. The incoming waves are then quantified by the LODI (Locally One Dimensional Inviscid) system which is directly derived from the Navier-Stokes equations written for primitives variables in characteristic form. Lodato et al. (2008) proposed to use the three directions of space to better describe oblique waves. Each method requires a special treatment for the first wave which comes from the outer to inner of the computational domain. 


\section{Entering wave amplitude term}

\subsection{Classic NSCBC}

First, let us focus on the LODI formulation for the "entering wave" $\mathcal{N}_{1}$. The wave amplitude is modified in order to make the boundary non-reflective. One can impose the amplitude as 0 but this will lead to an unconstraint density which is most generally unstable or conduct to drift in pressure at the boundary. Then the "perfectly non-reflecting" boundary condition would lead to stability issues due to the lack of informations on the mean density $\rho_{\infty}$ far from the computational domain. Therefore, this mean density can be imposed at the boundary but this treatment would generate reflected waves.

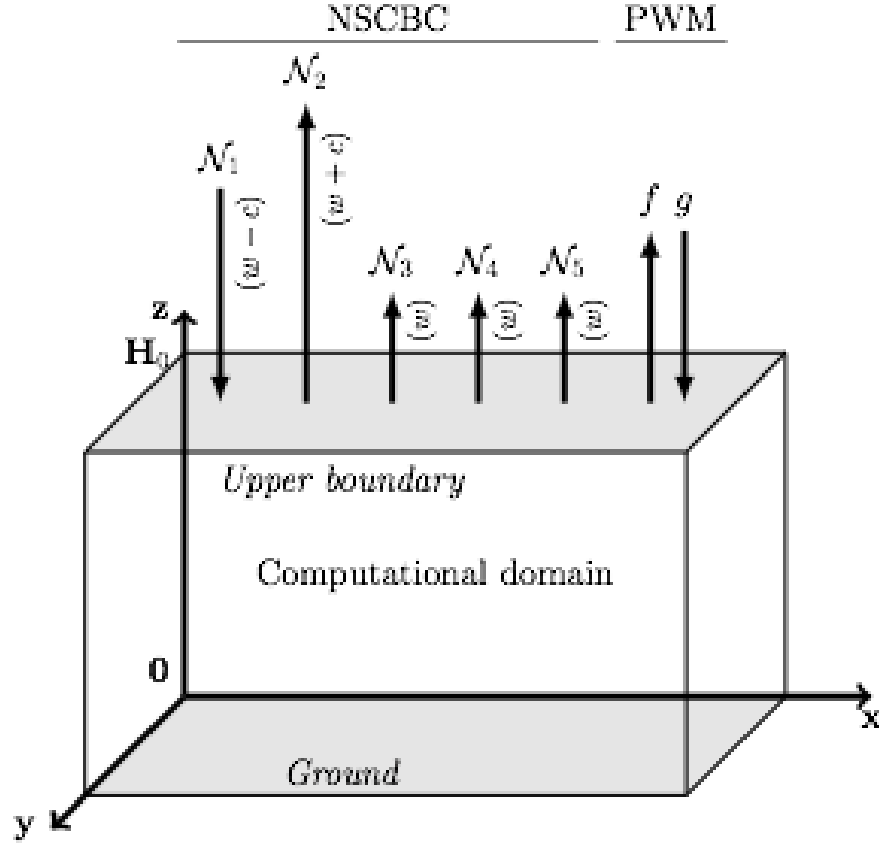

Figure 1 - Outgoing and entering waves through the upper boundary $\left(z=H_{0}\right)$ of the computational domain for classic NSCBC and PWM theories
In that case, we should add some physical informations on $\rho_{\infty}$ to the boundary in a "soft" way to avoid strong reflection. A simple way to do it is to set the entering wave as the linear relaxation term of Rudy and Strikwerda (1980)

$$
\mathcal{N}_{1}=K\left(\rho-\rho_{\infty}\right)
$$

where $K$ is a constant $\left[s^{-1}\right]$. When $K$ is set to 0 , we get back to the unstable "perfectly nonreflecting" boundary and when $K$ is big enough, the boundary is closer to the fully reflecting behavior. Selle et al. (2004) have studied the impact of the coefficient $K$ on the boundary reflection and have shown that the boundary is equivalent to a low-pass filter with $K / 4 \pi$ as cut off frequency. Nevertheless, in our problem, the Rayleigh top damping layer provides high frequency filtering, meaning that the new upper boundary shall only evacuate the remaining low frequency waves which can not be done with this theory.

\subsection{Plane Wave Masking}

An extension of the "classic" NSCBC presented above called PWM (Plane Wave Masking) is built to filter the low frequency waves. There, it is favorable to distinguish turbulent (" ' ") and acoustic (" $\sim$ ") perturbations of the flow variables $e . g$.

$$
\begin{aligned}
\rho_{d}(x, y, z, t) & =\overline{\rho_{d}}(x, y, z)+\rho_{d}^{\prime}(x, y, z, t)+\tilde{\rho}_{d}(z, t) \\
w(x, y, z, t) & =\bar{w}(x, y, z)+w^{\prime}(x, y, z, t)+\tilde{w}(z, t)
\end{aligned}
$$

Here again, the acoustic perturbations are assumed one-dimensional and normal to the boundary. For sufficiently small fluctuation amplitude, the linearized characteristics wave amplitudes are given by $f=f(z-(\bar{w}+\bar{c}) t)$ and $g=g(z-(\bar{w}-\bar{c}) t)$, travelling respectively in the positive and negative vertical direction. Using the acoustic fluctuation, one can write

$$
f=\frac{1}{2}\left[\frac{\tilde{P}}{\bar{\rho} \bar{c}}+\tilde{w}\right], \quad g=\frac{1}{2}\left[\frac{\tilde{P}}{\bar{\rho} \bar{c}}-\tilde{w}\right]
$$


It is then possible to adapt the classic NSCBC to get a non-reflecting boundary for low frequency waves. The idea of PWM is to identify outgoing waves at the boundary using the above equation and then explicitly eliminate outgoing wave contribution from the linear relaxation term.

$$
\mathcal{N}_{1}=K\left[\rho-\rho_{\infty}-\frac{\bar{\rho}}{\bar{c}} f\right]
$$

The "masked" outgoing wave no longer contributes to the "entering" wave $\mathcal{N}_{1}$ ans should leave the domain without any reflection.

\subsection{Reflection coefficient}

In order to evaluate the boundaries efficiency, it is important to properly define the reflection of the incoming wave. The reflection coefficient $r(\omega)$ is typically dependent of the acoustic incoming wave frequency (or pulsation). Basically, the reflection is the ratio between the reflected wave, seen as the "entering wave", amplitude ans the incoming wave amplitude. The incoming wave can be seen either as $\mathcal{N}_{2}$ or $f$. Therefore, the reflected wave becomes respectively $\mathcal{N}_{1}$ or $g$. Selle et al. (2004) define the reflection coefficient as

$$
r(w)=\frac{\mathcal{N}_{1}}{\mathcal{N}_{2}}
$$

whereas Polifke et al. (2006) use

$$
r(w)=\frac{\hat{g}}{\hat{f}} .
$$

The ".." denotes the Fourier transform of the quantity ". ". This last definition only stands if the coefficient $K$ is large enough so that the drift of density or velocity is effectively eliminated. Thus, deviations from the target density at the boundary will be controlled by the acoustic signal. This coefficient gives a first comparison point between the classic NSCBC and the PWM extension. According to Polifke et al. (2006), with $\tau \equiv 2 / K$,

The classic formulation is a low-pass whereas the plane wave masking extension allows, by construction, the radiation of the low-frequency waves. This study is only valid for harmonic plane waves of arbitrary non-zero frequency. This post-processing coefficient is useful to measure the efficiency of the boundary and this shows that the PWM should be the best solution as it is compliant at least with (a) and (c).

\section{MesoNH implementation}

The two solutions have been implemented in an off-line Python code to investigate the performances of the new boundary. This implementation has required several changes at the term computation method level but not on the philosophy of the process. Indeed, the characteristic boundary conditions have not been written in a stratified atmosphere and this hypothesis requires some adjustments. In this section, we will focus on 1D formulation, i.e. Eq. (14), with plane wave masking. Let us write the equations system really solved by MesoNH at the new boundary for the density $\rho$, the potential temperature $\theta$ and the vertical wind $w$. 


$$
\begin{array}{r}
\frac{\partial \rho}{\partial t}+\mathcal{N}_{1}+\mathcal{N}_{2}+\mathcal{N}_{3}=0 \\
\frac{\partial w}{\partial t}+\frac{c}{\rho}\left(\mathcal{N}_{2}-\mathcal{N}_{1}\right)+K_{3} \Delta_{z} w \\
\hdashline \frac{\partial \theta}{\partial t}-\frac{1}{\varphi} \mathcal{N}_{3}+\frac{\partial^{L} \theta}{\partial z}+K_{4} \Delta_{z} \theta
\end{array}
$$

The framed terms are added to the original system and will be detailed further. The wave amplitudes have also been modified from the original method.

$$
\begin{aligned}
& \mathcal{N}_{1}=K_{1}\left(\rho-\rho_{L S}\right)-K_{2} \frac{\rho_{L S}}{c_{L S}} f \\
& \mathcal{N}_{2}=\frac{w+c}{2}\left[\frac{\partial^{*} \rho}{\partial z}+\frac{\rho}{c} \frac{\partial^{*} w}{\partial z}+\varphi \frac{\partial^{*} \theta}{\partial z}\right] \\
& \mathcal{N}_{3}=-w \varphi \frac{\partial^{*} \theta}{\partial z}
\end{aligned}
$$

Several main terms have been changed so let us have a look on each modification and theirs consequences.

\subsection{Background stratification and hydrostatic equilibrium}

The atmosphere is a vertically stratified domaine due to the hydrostatic equilibrium which can be described by

$$
\frac{\partial P}{\partial z}+\rho g=0 .
$$

The stratification directly implies that the spacial derivative computed at the boundary for the NSCBC, with or without PWM extension, are non-zero even in equilibrium state. If the original system was implemented without any modifications, the hydrostatic state would have been treated as a fluctuation leading to an unstable boundary. Then, the first step is to split the hydrostatic part and the perturbational part of the signal. For this purpose, we uses the large scale fields for the density, the wind and the potential temperature. They are provided by another simulation from a larger scale model as AROME. This previous simulation has already computed fields at the boundary taking into account the hydrostatic equilibrium. Thus, the main variables are written using the large scale fields denoted by the subscript "LS" and a perturbation term denoted by " $\sim$ ".

$$
\begin{array}{lll}
\rho=\rho_{L S}+\tilde{\rho}, & w=w_{L S}+\tilde{w} \\
\theta=\theta_{L S}+\tilde{\theta}, & P=P_{\infty}+\tilde{P}
\end{array}
$$

The pressure $P$ is not provided by the large scale model and its reference state should be computed using other variables which have a large scale field available. As the fluctuations of pressure are an important part of the PWM extension, a specific attention should be taken to define its reference state (see section 4.4). Therefore, this notation allows to create two new derivative operators related to each term.

$$
\begin{aligned}
\frac{\partial^{L S} \star}{\partial z} & \equiv \frac{\partial \star_{L S}}{\partial z} \\
\frac{\partial^{*}}{\partial z} & \equiv \frac{\partial \star}{\partial z}-\frac{\partial^{L S} \star}{\partial z} \\
\star & =\{\rho, w, \theta\}
\end{aligned}
$$


The new derivative operators takes into account the whole hydrostatic equilibrium and then the gravity term which can be found in $D$ in Eq. (14) is no longer relevant and does not appear in Eq. (26).

\subsection{Potential temperature transport}

The potential temperature should be advected at the wind velocity $w$ in the Eq. (27). Without any compensation, framed terms in Eq. (27), the boundary would have advected the perturbation state only as it is reduced to

$$
\frac{\partial \theta}{\partial t}+w \frac{\partial^{*} \theta}{\partial z}=0
$$

By adding the large scale field transport, $w \frac{\partial^{L S} \theta}{\partial z}$, to Eq. (36) and using the definition of operators in Eq. (35), it becomes

$$
\begin{aligned}
& \frac{\partial \theta}{\partial t}+ w \frac{\partial^{*} \theta}{\partial z}+w \frac{\partial^{L S} \theta}{\partial z}=0 \\
& \Longleftrightarrow \frac{\partial \theta}{\partial t}+w \frac{\partial \theta}{\partial z}=0
\end{aligned}
$$

This new term balance the advection of the total potential temperature which is coherent with the overall transport equation - Eq. (4).

The last two terms in Eq. (26) and Eq. (27) are designed to stabilize the overall boundary behavior, i.e. $\frac{\partial^{*} \theta}{\partial z} \underset{t \rightarrow \infty}{\rightarrow} 0$ and $\frac{\partial^{*} w}{\partial z} \underset{t \rightarrow \infty}{\rightarrow} 0$. They will not going to be detailed further in this study but it is shown that they do not affect the dynamic behavior of the boundary which is the most important when radiating waves.

\subsection{PWM extension formulation}

In this section, we will focus on the formalism used to compute the "entering wave" $\mathcal{N}_{1}$. There is two main parts in this term as shown Eq. (28). The first one corresponds to the classic linear relaxation term of (Rudy and Strikwerda 1980) but the reference state used is the large scale density. The second one is the plane wave masking expression. Using Eq. (19) and Eq. (32-33), we set

$$
f=\frac{1}{2}\left[\frac{\tilde{P}}{\rho_{L S} c_{L S}}+\tilde{w}\right]
$$

Here, the definition of $P_{\infty}$ is very important and it can lead to a significant error of $\rho(t \rightarrow \infty)$. The pressure is a diagnostic variable computed by

$$
P=P_{00}^{1-\gamma}\left[\rho R_{d} \theta\right]^{\gamma},
$$

where $P_{00}$ is a reference value of the pressure and $R_{d}$ the gas constant for dry air. A natural way to define $P_{\infty}$ with large scale fields is

$$
P_{\infty}=P_{00}^{1-\gamma}\left[\rho_{L S} R_{d} \theta_{L S}\right]^{\gamma} \text {. }
$$

Therefore, the used definition of the reference pressure is

$$
P_{\infty}=P_{00}^{1-\gamma}\left[\rho_{L S} R_{d} \theta\right]^{\gamma} \text {. }
$$

The onl y difference between the expression is the use of the total potential temperature instead of the large scale one. 


\subsection{Drift in mean density depending on the reference pressure definition}

In order to show the consequences of such a choice, let us consider the steady state reached after a wave radiation. Each temporal derivative is null in Eq. (25-27). For the sake of clarity, $K_{3}=K_{4}=0$ which means that no stability correction is applied. Moreover, in general case, the large scale potential temperature profile is not constant, i.e. $\frac{\partial^{L S} \theta}{\partial z} \neq 0$. On the another hand, we assume that the wave had changed the local equilibrium of the potential temperature as $\tilde{\theta} \neq 0, \frac{\partial^{*} \theta}{\partial z}=0$. The last hypothesis is that the large scale vertical wind is null, i.e $w_{L S}=0$. Under those hypothesis, we have necessarily $w=$ $w=0$ in order to satisfy Eq. (27). The wave amplitude $\mathcal{N}_{3}$ is also null due to the local constant vertical

profile of the potential temperature fluctuation. Finally, the system is reduced to two equations

$$
\begin{aligned}
& \mathcal{N}_{1}+\mathcal{N}_{2}=0, \\
& \mathcal{N}_{2}-\mathcal{N}_{1}=0 .
\end{aligned}
$$

This system is equivalent to

$$
\mathcal{N}_{1}=\mathcal{N}_{2}=0 \text {. }
$$

For $\mathcal{N}_{2}$, it can be seen as each perturbation field is locally, at least, constant over $z$ which is quite good for a steady state. Thus, the only tricky equation is

$$
\mathcal{N}_{1}=K_{1} \tilde{\rho}-K_{2} \frac{\rho_{L S}}{c_{L S}} f=0 \text {. }
$$

With $c_{L S}=\frac{\gamma P_{L S}}{\rho_{L S}}$, we have

$$
K_{1}\left[\frac{\rho}{\rho_{L S}}-1\right]=\frac{K_{2}}{2 \gamma}\left[\frac{P}{P_{L S}}-1\right] .
$$

At this stage, the definition of the reference pressure becomes important. If the Eq. (40) is used, the ratio $\alpha=\left[\frac{\theta}{\theta_{L S}}\right]^{\gamma}$ will appear and we can show that the deviation on density at the steady state is

$$
\frac{\tilde{\rho}}{\rho_{L S}}=\frac{1}{\gamma} \frac{K_{2}(1-\alpha)}{\alpha K_{2}-2 K_{1}} .
$$

This ration is zero if $K_{2}=0$ or $\alpha=1$. The first solution gives a exact steady state if the used method is NSCBC without plane wave masking. This solution is not satisfying because PWM is necessary to ensure low-frequency waves radiation. In the other solution, the ratio $\alpha$ should be unity which is in contradiction with the baseline assumption of $\theta \neq 0$. Therefore, this definition of $P_{\infty}$ leads to inaccuracy of the density steady state.

On the other hand, if the Eq. (41) is used, the ratio $\alpha$ does not appear - equivalent to always unity - which leads to an accurate boundary. Those behavior have been verified numerically but the results are not presented here and for following simulations, the Eq. (41) will always be used as the reference pressure.

\section{Simulation results}

Full compressible simulation of a gaussian density perturbation has been performed to validate the non-reflective character of the new boundary. The initial condition is the hydrostatic state, here equivalent to the large scale state, to which a density perturbation is added as 


$$
\rho(z, t=0)=\rho_{L S}(z)+\rho_{a} \exp \left[-\frac{\left(z-\frac{H_{0}}{2}\right)^{2}}{d_{a}^{2}}\right],
$$

where $\rho_{a}$ is the perturbation amplitude $\left[\mathrm{kg} \cdot \mathrm{m}^{-3}\right], d_{a}$ the characteristic spreading $[\mathrm{m}]$ of the initial perturbation and $H_{0}$ the height of the domain. The ground is set as slip wall and other boundaries are cyclic. The cells are $50 \mathrm{~m}$ long in each direction and the grid is $7 \times 3 \times 102$ in $x, y, z$ direction respectively. The problem is actually quasi $1 \mathrm{D}$ at this point. This time step used is 0.05 s. No turbulence, relaxation and Rayleigh damping layer was added to look a the boundary efficiency without any help of other methods.

The wind advection scheme used is $4^{\text {th }}$ order centered scheme used with RKC4 scheme, the scalar advection scheme is PPM in its monotonic and positive definite version. The numerical viscosity added to the momentum equation is set to $3 \cdot 10^{3}[\mathrm{~Pa} \cdot \mathrm{s}]$. The Brunt-Väisälä frequency, defined as $N=\sqrt{\frac{g}{\theta} \frac{d \theta}{d z}}$, is set to $0.02\left[s^{-1}\right]$.

\subsection{Results for one-dimensional wave}

For the simulations discussed in this section, the density perturbation is set to $\rho_{a}=0.01\left[\mathrm{~kg} \cdot \mathrm{m}^{-3}\right]$ and $d_{a}=300[\mathrm{~m}]$. The wind is set to zero everywhere so only the acoustic signal will generate wind. The stability coefficient are set to $K_{3}=0\left[\mathrm{~s}^{-1}\right], K_{4}=0.1\left[\mathrm{~s}^{-1}\right]$. The relaxation coefficient $K_{1}$ is set to $0.1\left[\mathrm{~s}^{-1}\right]$ which allows no drift from the large scale density. For the NSCBC simulation, $K_{2}=0\left[\mathrm{~s}^{-1}\right]$ and for the plane wave masking simulation, it is equal to $K_{1}$. Both simulation are compared with the old boundary condition, denoted $\mathrm{MNH}$, which forces $w=0$ at the top and computes othe $\mathrm{r}$ quantities with hydrostatic equilibrium equation.

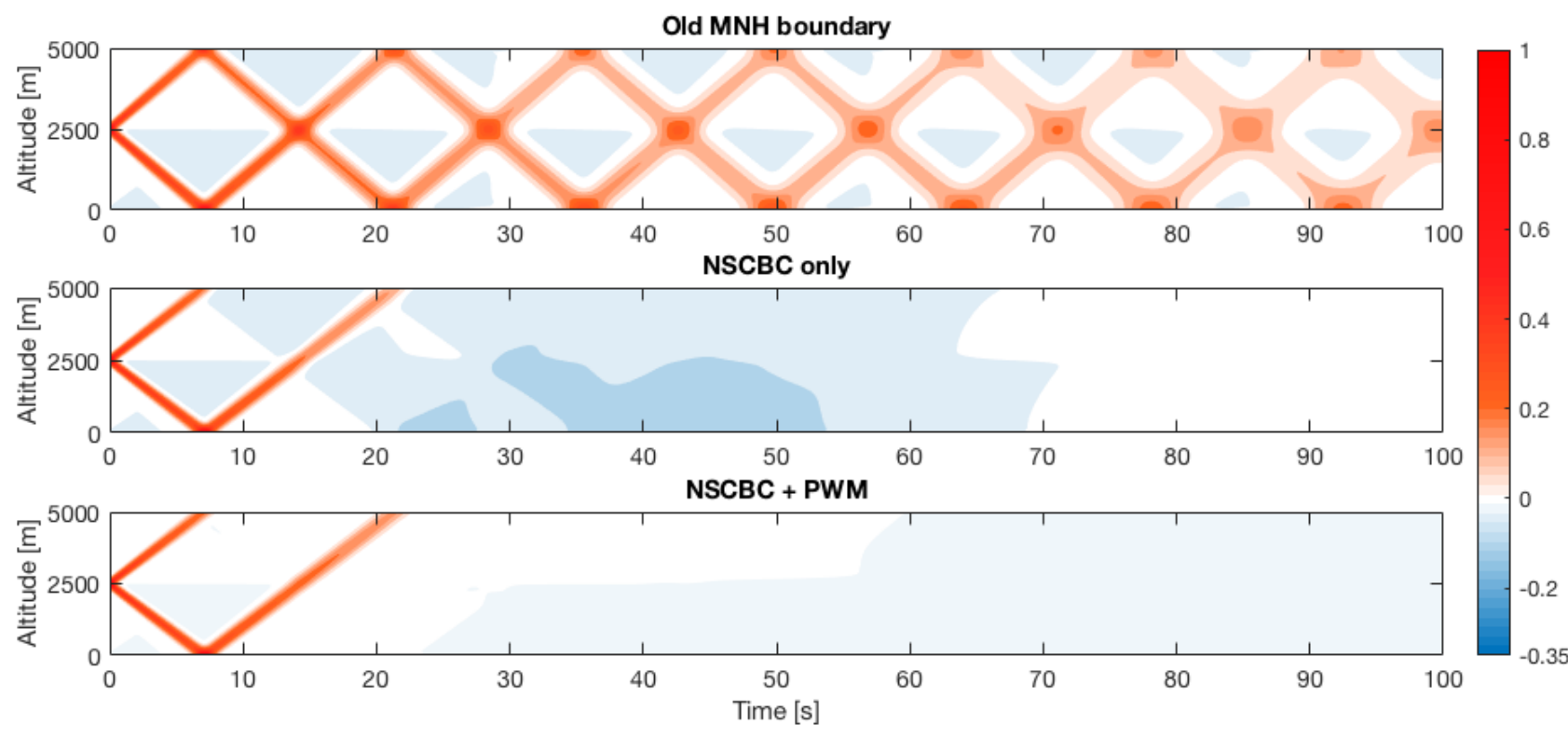

Figure 2 - Relative density anomaly [-] for the different boundaries. CI : 0.05

The relative density anomaly is defined by

$$
\tilde{\rho}^{*}=\frac{\tilde{\rho}}{\rho_{a}}=\frac{\rho-\rho_{L S}}{\rho_{a}} .
$$




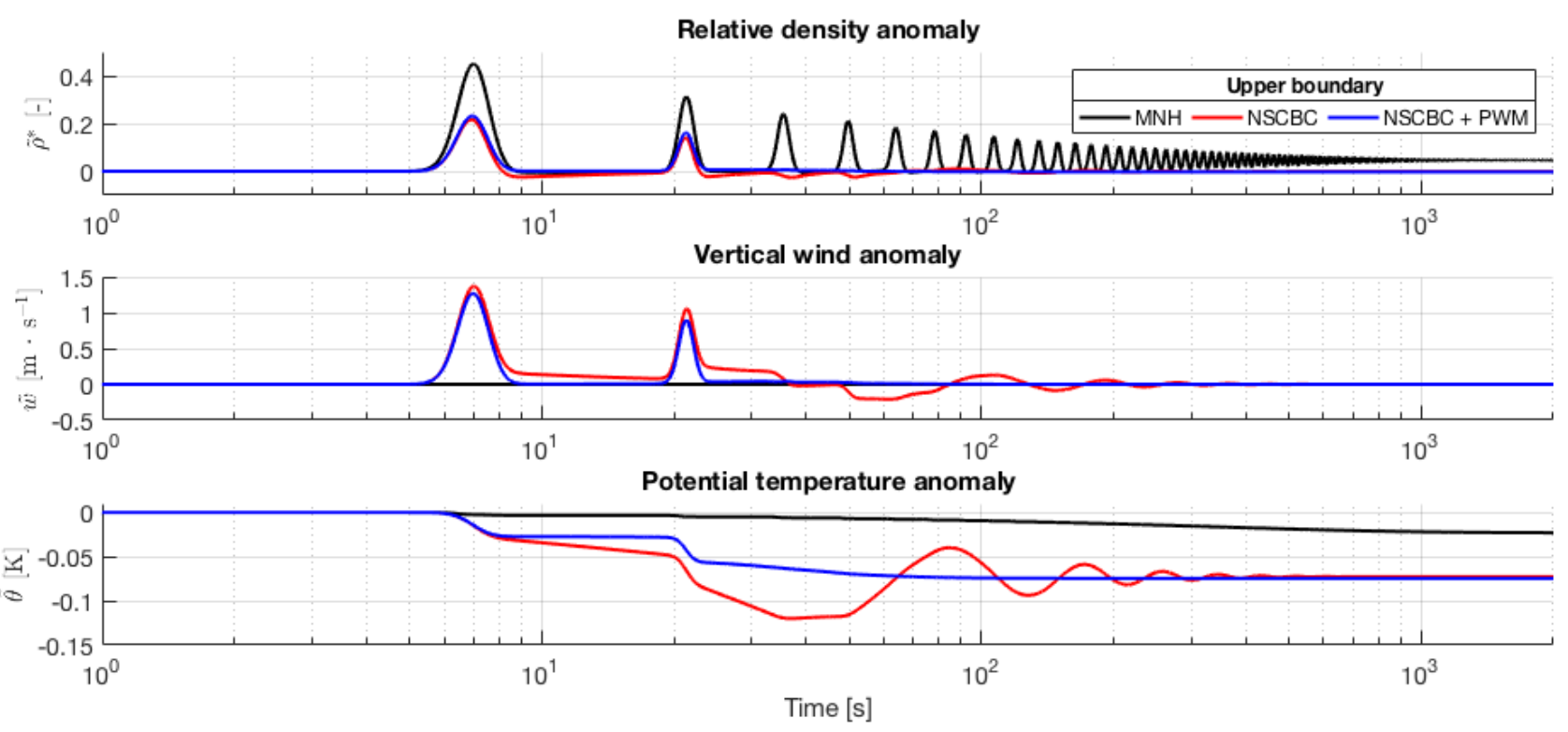

Figure 3 - Main fields anomaly over time at the upper boundary for the different methods for 1D gaussian wave perturbation

As shown Fig. 2, the old boundary reflects the whole signal and the numerical viscosity diffuses it through time, that is why the amplitude of the relative density anomaly decreases and its spreading increases. The behavior is completely non satisfying because of the perturbation near the ground that were reflected on the upper boundary. The NSCBC does a quite good job with this coefficient but their is still an important reflection which is not compliant enough with the first criterion (a). The plane wave masking extension does a great improvement in radiating the waves as no reflection occurs. In order to better understand and quantify the dynamic and static behavior of each boundaries, the difference fields are studied at the boundary.

In Fig. 3, the static behavior is reached around 1000s for both MNH and NSCBC boundaries whereas the PWM extension strikes the balance in $400 \mathrm{~s}$. Moreover, the final state is the same for NSCBC, with and without PWM, which is consistant with the study in section 4.4. The new boundary converges to zero anomaly for both wind and density and advects the potential temperature in the same way that the inner domain, i.e. the vertical gradient of $\theta$ is zero. On the other hand, the dynamic behavior is the capability to radiate efficiently an incoming wave. The MNH boundary is not good at this exercice as the wind is strictly zero at the boundary, which explain the low advection of the potential temperature. The waves are constantly bouncing on the boundaries while the numerical diffusion weakens the wave amplitude. The NSCBC boundary is more efficient but is not perfect as some residual wind and density anomaly are still existing after the wave passage across the domain limit. Therefore, some oscillations of the wind and consequently on potential temperature are induced in the domain. The plane wave masking extension fixes those problems in changing the dynamic behavior of the boundary which leads to zero residual density and wind anomalies after the wave crossing.

The overall performance of the boundaries can also be shown on the reflection coefficient. On Fig. 4 , the plane wave masking gets near zero reflection for low-frequencies as predicted. The classic NSCBC is partially non-reflecting and is partially compliant with the requirements for the new boundary. 


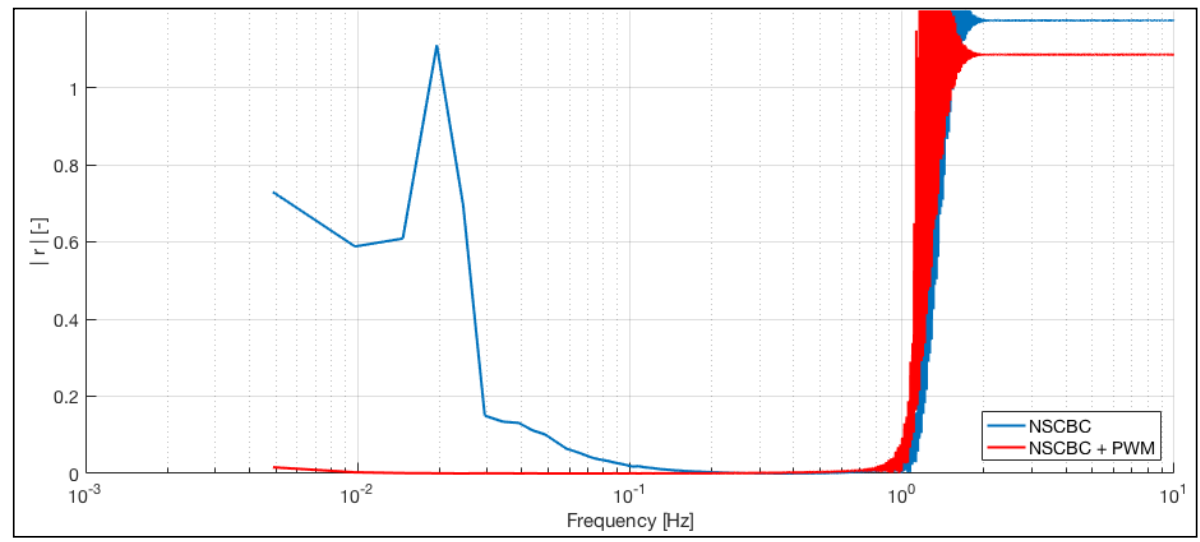

Figure 4 - Reflection coefficient for one-dimensional wave without and with plane wave masking

\subsection{Results for spherical wave}

The gris used is now $326 \times 3 \times 102$ in $x, y, z$ directions respectively. Cell size, time step, temporal and spacial schemes are the same that the ones used in section 5.1. The initial perturbation formulation is now two dimensional in order to test the boundary for non-normal incoming waves.

$$
\rho(z, t=0)=\rho_{L S}+\rho_{a} \exp \left[-\frac{\left(z-\frac{H_{0}}{2}\right)^{2}+\left(x-\frac{L_{0}}{2}\right)^{2}}{d_{a}^{2}}\right]
$$

Fig. 5 shows that the new boundary radiates the spherical wave as well as the normal wave. The two top images show the wave expansion before the boundary interaction. The bottom left image points the reflection on the left part and the wave passing through on the right side. At that moment, the incoming wave reaches the boundary with normal direction, so the behavior is consistant with the one dimensional study. The bottom right image show the strong reflection for the old boundary condition and the correct radiation for the new boundary even if the wave comes with an oblique direction. The sphericity is not affect by the local 1D hypothesis at the boundary.

\section{Conclusion and prospects}

The new boundary implemented in the compressible version of Meso-NH has shown great interests in evacuating acoustic waves in order to get a better precision near of the ground. To allow waves to go out of the domain, the NSCBC theory with the plane wave masking layer has been implemented and tested for planar, oblique and spherical waves. Its behavior has also been checked for various academic and classic Meso-NH cases as hydrostatic and non-hydrostatic orographic waves as well as fire experiments. Then, the new boundary is compliant with all of the criterions given in section $\mathbf{1}$. The new boundary should lead to a better accuracy near of the fire front for futur simulations. The compressible assumption will be evaluated with the FireFlux experiment (Filippi et al. 2013) and compared to results already gathered by the anelastic version of Meso- $\mathrm{NH}$ on Aullene fire in Corsica (Filippi et al. 2018). 

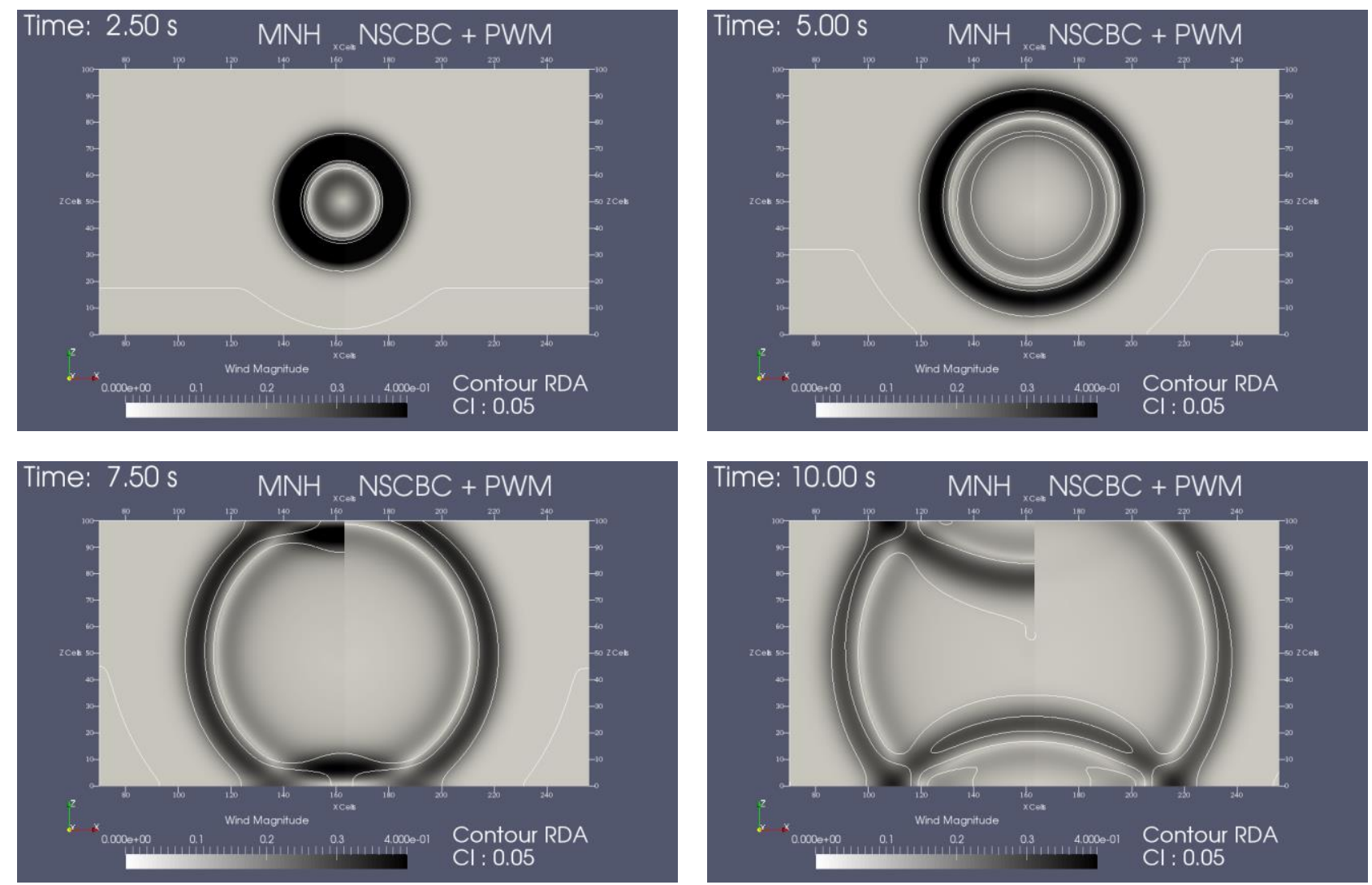

Figure 5 - Comparison of spherical wave radiation for MNH (left part of each image) and NSCBC + PWM (right part of each image). Wind magnitude is presented in colored contour and Relative Density Anomaly (RDA) in white line contour for $-0.05,0$ and 0.05 [-].

\section{References}

Colonius, T. (2004). Modeling Artificial Boundary Conditions for Compressible Flow. Annual Review of Fluid Mechanics, 36(1), 315-345.

Filippi, J.-B., F. Bosseur, C. Mari, C. Lac, P. Le Moigne, B. Cuenot, D. Veynante, D. Cariolle, and J. H. Balbi, (2009). Coupled atmosphere-wildland fire modelling, J. Adv. Model Earth Syst., 1, \#11.

Filippi, J.-B., X. Pialat, and C. G. Clements, (2013). Assessment of ForeFire/Meso-NH for wildland fire/atmosphere coupled simulation of the FireFlux experiment, Proc. Combustion Inst., 34, 26332640.

Filippi, J.-B., Bosseur, F., Mari, C., \& Lac, C. (2018). Simulation of a large wildfire in a coupled FireAtmosphere model, Atmosphere, 9(6), 218.

Lac, C., J.-P. Chaboureau, V. Masson, J.-P. Pinty, P. Tulet, J. Escobar, M. Leriche, and co-authors (2018). Overview of the Meso-NH model version 5.4 and its applications, Geosci. Model Dev., p. in discussion.

Lafore, J. P., J. Stein, N. Asencio, P. Bougeault, V. Ducrocq, J. Duron, C. Fischer, P. Hereil, P. Mascart, J. P. Pinty, J. L. Redelsperger, E. Richard, and J. Vila-Guerau de Arellano, (1998). The Meso-NH Atmospheric Simulation System. Part I: Adiabatic formulation and control simulations. Annales Geophysicae, 16, 90-109.

Lodato, G., Domingo, P., \& Vervisch, L. (2008). Three-dimensional boundary conditions for direct and large-eddy simulation of compressible viscous flows. Journal of Computational Physics, 227(10), 5105-5143. 
Polifke, W., Wall, C., \& Moin, P. (2006). Partially reflecting and non-reflecting boundary conditions for simulation of compressible viscous flow. Journal of Computational Physics.

Poinsot, T. J., \& Lele, S. K. (1992). Boundary conditions for direct simulations of compressible viscous flows. Journal of Computational Physics, 101(1), 104-129.

Rudy, D. H., \& Strikwerda, J. C. (1980). A nonreflecting outflow boundary condition for subsonic navier-stokes calculations. Journal of Computational Physics, 36(1), 55-70.

Selle, L., Nicoud, F., \& Poinsot, T. (2004). Actual Impedance of Nonreflecting Boundary Conditions: Implications for Computation of Resonators. AIAA Journal, 42(5), 958-964.

Thompson, K. W. (1987). Time dependent boundary conditions for hyperbolic systems. Journal of Computational Physics, 68(1), 1-24. 